\title{
Implementation of Micro-Electro-Mechanical Systems for Broadband Folded Dipole Array
}

\author{
Jiang Chang ${ }^{1, a}$, Fang Wei ${ }^{1, b}$ \\ ${ }^{1}$ The School of Higher Vocational Education, University of Science and Technology Liaoning, 185 \\ Qianshan Zhong Road, Anshan, Liaoning, China, 114051 \\ aemail: asweifang@126.com, bemail:aschangjiang@126.com
}

Keywords: MEMS; Radio Frequency Communication; Space Diversity

\begin{abstract}
Micro-Electro-Mechanical Systems (MEMS) are widely used in biotechnology, medicine, communication and inertial sensing. Utilizing the integrated combination of electro and mechanical systems, MEMS are able to achieve new functionality. In particular, a folded dipole array is an important component in radio frequency communication due to its broadband feature. One major drawback preventing folded dipole arrays being used in certain applications lies in their unidirectional nature, i.e., their radiation direction is fixed once the fabrication is done and not easy to change in use. In this paper, we propose a novel method to implement MEMS for a folded dipole array, which makes the radiation direction flexible. A comparison of whether MEMS are implemented on radiating elements to make them move or as circuit elements is also reported to provide insight for future research in the field of radio frequency MEMS.
\end{abstract}

\section{Introduction}

There is no universal definition of micro-electro-mechanical systems (MEMS) in the world. However, it is generally agreed that MEMS should possess the following features: (i) the size of MEMS should be small (Typically, the critical physical dimension is from micrometer to millimeter.); (ii) in MEMS, there should be both electro and mechanical elements (It is not necessary for MEMS to be able to move.); (iii) MEMS should be able to realize a certain function that neither solely electro systems nor mechanical systems can achieve; (iv) MEMS are produced with techniques of micro-fabrication (e.g., bulk micromachining, surface micromachining and high aspect ratio silicon micromachining).

MEMS are widely used in many engineering domains. Some common applications include inkjet printers (piezoelectric bubble ejection is used to deposit ink on paper), and accelerometers in modern cars (for airbag deployment in collision, e.g.) and drones (for yaw stabilization, e.g.). In biotechnology, MEMS enables biochips for detection of hazardous chemical and biological agents. The very domain that benefits most from MEMS could be medicine. Pressure sensors are probably the most successful application of MEMS in medicine, at least considering the number of devices manufactured.

In this work, we focus on MEMS in radio frequency communication, which offer enhanced performance over the conventional radiating elements (e.g., antenna arrays) and solid-state devices (e.g., semiconductor switches and variable capacitors) [1]. The rest of this paper is organized as follows: we first discuss two types of MEMS in radio frequency communication - MEMS in radiating elements to make them movable and MEMS as circuit elements to alter the electrical length. Advantages and disadvantages of both types are compared. Then, a novel method to implement MEMS for a folded dipole array is reported, which addresses the major drawback of folded dipole arrays, i.e., the inflexible radiation direction. Lastly, concluding remarks of the paper are made and future research work is briefly mentioned.

\section{MEMS in Radio Frequency Communication}

As discussed above, there are two ways to implement MEMS in radio frequency communication. The first way is implementing movable radiating elements. There have been many successful examples. The first one was built over a decade ago [2], where each radiating arm of a vee antenna 
is capable of independent movement, giving the possibility of far-field beam steering and beam shaping. As a result, the azimuthal pointing direction and directivity of the main beam can be controlled independently. Other candidates for this type of MEMS implementation include Quasi-Yagi antenna arrays [3].

Advantages of implementing MEMS on radiating elements are obvious. Radiation patterns become adaptive and high reliability can be achieved. However, there are also challenges to address. First, radiating elements are sensitive to the presence of nearby structures. Therefore, the interactions resulting from moving might distort expected radiation patterns. Second, for high-frequency communication (e.g., millimeter wave), antennas have short wavelengths, which are comparable to the physical dimensions of adjacent structures, increasing the risk of strong resonant-mode coupling. Third, for low-frequency communication, if radiating elements are detached from the substrate, the size-reducing attribute offered by the non-air dielectric cannot be exploited. This could make the antennas too large.

The second way to implement MEMS in radio frequency communication is through circuit elements. For instance, switches and variable capacitors can be added to alter the electrical length. There have also been many successful examples. Simple dual-frequency reconfigurable slot dipole arrays for X- and Ka-band are reported in [4]. A single-arm spiral antenna with RF MEMS switches located along the line is reported for X-band operation in [5]. Folded antennas capable of operation in five mobile phone bands are realized using radio frequency MEMS solutions [6]. An example is illustrated in Fig. 1. Switches in the slot are used to adapt the electrical length, and thus the operating band.

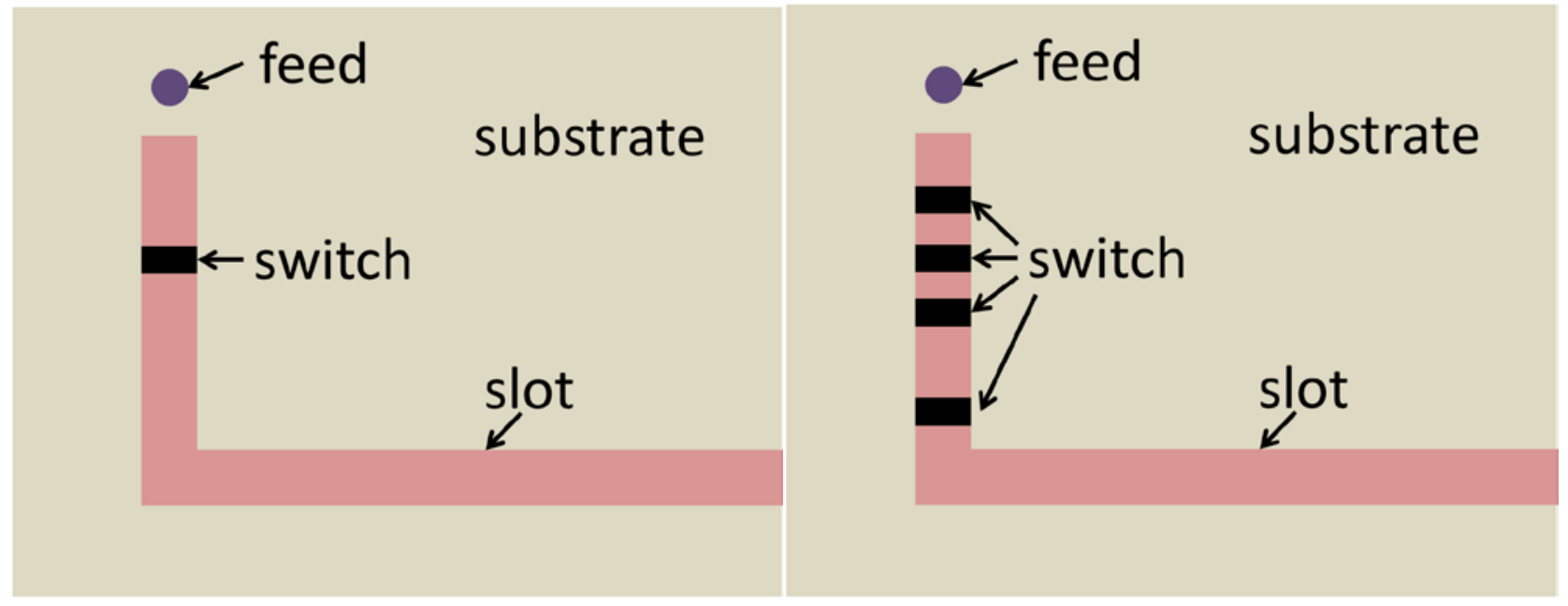

(a) One switch exists in the slot

(b) Multiple switches exist in the slot

Fig. 1. An example of MEMS as circuit elements in radio frequency communication

Advantages of implementing MEMS through circuit elements are mainly in two aspects. First, multi-band behavior can be achieved. Second, space diversity can be obtained. The major drawback is in reliability [7]. It is very challenging to make these devices (e.g., switches and variable capacitors) perform reliably. Or in more general terms, reliability of almost all engineering products is not easy to achieve. Mechanisms like hot-carrier instability (HCI) and negative-bias temperature instability (NBTI) could cause failure of the entire system. Reliability issues are more severe as the size of the device becomes smaller. Therefore, MEMS must be carefully designed, taking reliability into account. Extensive tests are often required in order to address the reliability issue adequately. Comparison of these two types of MEMS implementations in radio frequency communication is presented in Table 1. 
Table 1. Comparison of two types of MEMS implementations in radio frequency communication

\begin{tabular}{|c|l|l|}
\hline MEMS Type & \multicolumn{1}{|c|}{ Radiating Elements } & \multicolumn{1}{c|}{ Circuit Elements } \\
\hline Feature & $\begin{array}{l}\text { Radiating elements are made } \\
\text { movable. }\end{array}$ & $\begin{array}{l}\text { Switches or variable } \\
\text { capacitors are inserted. }\end{array}$ \\
\hline Advantages & $\begin{array}{l}\text { Radiation patterns are } \\
\text { adaptive. } \\
\text { High reliability is achieved. }\end{array}$ & $\begin{array}{l}\text { Multi-band behavior is } \\
\text { achieved. } \\
\text { Space diversity is obtained. }\end{array}$ \\
\hline Challenges & $\begin{array}{l}\text { Radiation patterns can be } \\
\text { distorted. } \\
\text { Resonant coupling and large } \\
\text { size. }\end{array}$ & provide. \\
\hline Ease of Implementation & \multicolumn{1}{|c|}{ Easy } & \multicolumn{1}{|c|}{ Difficult } \\
\hline
\end{tabular}

\section{MEMS for Folded Dipole Array}

Folded dipole arrays are well known for their broadband behavior [8] [9]. However, the radiation is unidirectional, which prevents them from being used in certain applications. For example, in a military vehicle deployed to search enemy signals, an antenna array fixed onboard with one radiation direction is not very useful. We address this problem with a novel implementation of MEMS in a folded dipole array, as shown in Fig. 2 and Fig. 3. An axle is built through the center transmission line, so that the array is able to rotate around it. A light-weight electric motor is used to provide the driving force. The cross layer of the implementation is illustrated in Fig. 3. With this implementation, the radiation direction of the folded dipole array becomes adaptive and can be changed in use, depending on the real-time situation.

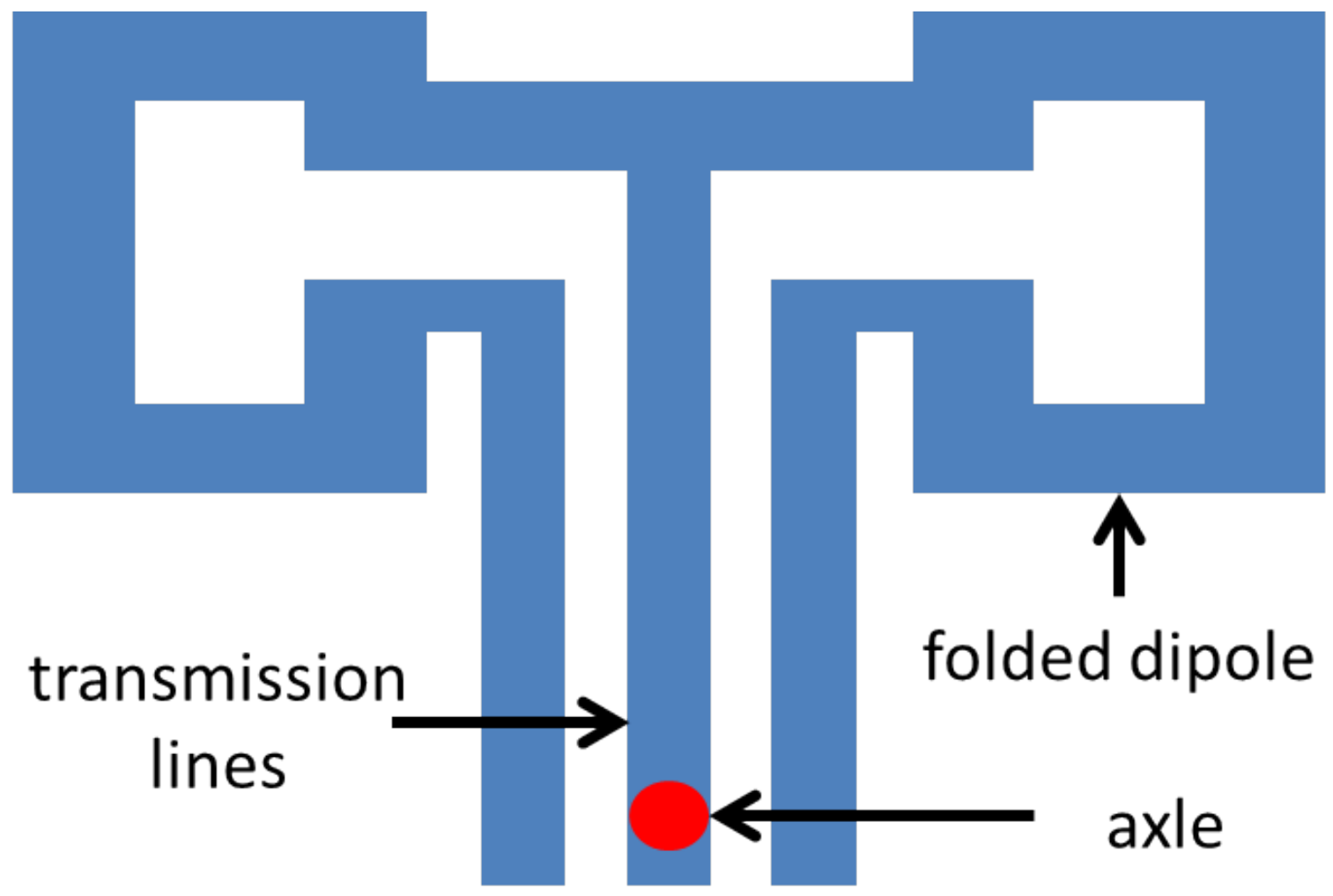

Fig. 2. Implementation of MEMS in a folded dipole array 


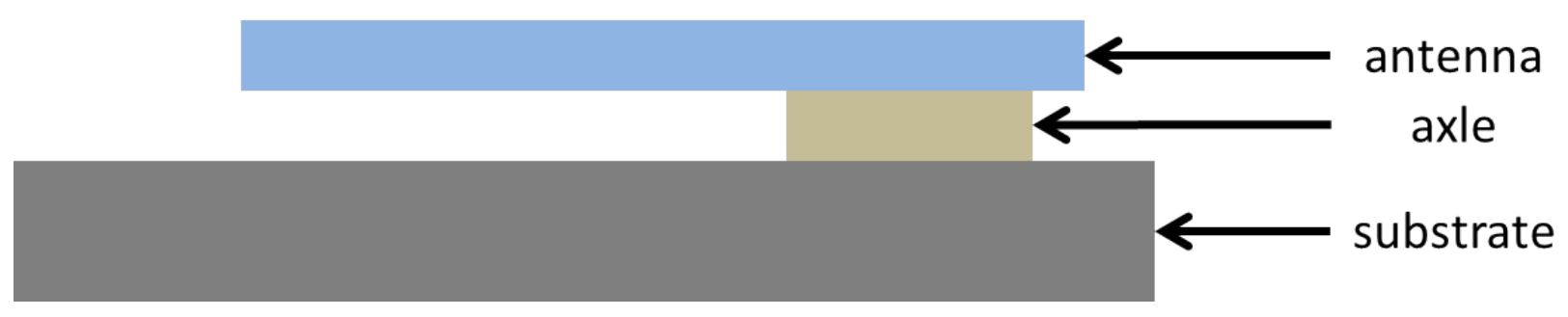

Fig. 3. Cross layer of the MEMS implementation

\section{Conclusion and Future Work}

In this paper, we present a novel implementation of a folded dipole array in radio frequency communication to achieve flexible radiation directions. A comparison of whether MEMS are implemented on radiating elements to make them move or as circuit elements is reported to provide insight for future research in the field of radio frequency MEMS. Real-time precise control of the rotation still needs to be figured out.

\section{References}

[1] Stepan Lucyszyn, Suneat Pranonsatit, RF MEMS for Antenna Applications, the $7^{\text {th }}$ European Conference on Antennas and Propagation (EUCAP), pp. 1988-1992, 2013.

[2] Jung-Chih Chiao, Yiton Fu, Lao Mak Chio, M. Delisio, Lih-Yuan Lin, MEMS Reconfigurable Vee Antenna, IEEE MTT-S International Microwave Symposium Digest, pp. 1515-1518, 1999.

[3] Wanli Chang, An Integrated W-Band High-Performance Quasi-Yagi Antenna Array, IEEE Antennas and Propagation Society International Symposium (APSURSI), 2010.

[4] K. Topalli, O. Civi, S. Demir, K. Sencer, T. Akin, Dual-Frequency Reconfigurable Slot Dipole Array with a CPW-based Feed Network Using RF MEMS Technology for X- and Ka-Band Applications, IEEE Antennas and Propagation Society International Symposium, pp. 825-828, 2007.

[5] Chang Won Jung, Ming-Jer Lee, G. Li, F. Flaviis, Reconfigurable Scan-Beam Single-Arm Spiral Antenna Integrated with RF-MEMS Switches, IEEE Transactions on Antennas and Propagation, Vol. 54, Iss. 2, pp. 455-463, 2006.

[6] K. Boyle, P. Steeneken, A Five-Band Reconfigurable PIFA for Mobile Phones, IEEE Transactions on Antennas and Propagation, Vol. 55, Iss. 11, pp. 3300-3309, 2007.

[7] Wanli Chang, Jiayu Luo, Yu Qi, Bo Wang, Reliability and Failure Analysis in Designing a Typical Operation Amplifier, $17^{\text {th }}$ IEEE International Symposium on the Physical and Failure Analysis of Integrated Circuits (IPFA), 2010.

[8] Wanli Chang, Jiayu Luo, 60-GHz Broadband Folded Dipole Array, International Workshop on Antenna Technology (iWAT), 2010.

[9] Wanli Chang, Jiayu Luo, Yuki Kawakami, Jinfeng Lin, A Novel Multi-Band Frequency Selective Surface Design and Its Application in a Compact 60-GHz Folded Dipole Array, International Conference on Electrical Engineering/Electronics Computer Telecommunications and Information Technology (ECTI-CON), pp. 1145-1149, 2010. 